\title{
De l'empâtement de la conscience à la psychopathologie (et retour)
}

\author{
Par HUBERT WYKRETOWICZ \\ CHUV / UNIL Lausanne / Université de Liège
}

Dans ce texte, j'entends considérer l'expression «pathologie de la liberté » dans un sens existentiel. Je mets donc entre parenthèses le sens social et politique de l'aliénation, tel qu'on peut le trouver par exemple sous la plume d'Honneth, afin de souligner la dimension existentielle du phénomène de l'aliénation.

Pour ce faire, je propose de considérer la philosophie sartrienne qui, comme on le sait, fait de la liberté le pivot de ses analyses dans L'Être et le Néant. Toutefois, je n'entends pas réactiver ici l'antique débat entre le déterminisme et la liberté, tel qu'on le trouve par exemple dans la quatrième partie de L'Être et le Néant. J'aimerais plutôt prendre pour fil conducteur cet étonnant phénomène de «l'empâtement » qui revient à plusieurs reprises sous la plume de Sartre et qui présente, à mon sens du moins, une ressource intéressante pour la psychopathologie phénoménologique, voire au-delà. Mon ambition est très simple : souligner un aspect de l'anthropologie philosophique de Sartre, et en indiquer la fécondité pour la psychopathologie. Je présuppose donc, au moins à titre de point de départ, que l'empâtement peut être considéré comme une forme pathologique de la liberté, autrement dit une forme d'aliénation de notre rapport au monde et à soi.

\section{La liberté sartrienne}

Il est archi-connu que la liberté sartrienne équivaut au pouvoir de néantisation de la conscience. Toutefois, je n'ai pas l'impression qu'on soit toujours bien 
au clair sur ce qu'il faut entendre exactement par là. Une version vulgarisée a tendance à faire de cette néantisation le privilège des décisions engagées du sujet sartrien, libre parce qu'engagé, libre parce que, quoi qu'il arrive, il est condamné à se décider, parce qu'on ne peut pas ne pas décider. Probablement qu'il y a du vrai dans cette vision activiste et volontariste de l'existentialisme sartrien, dont on trouverait la source dans l'Entschlossenheit heideggerienne ${ }^{1}$, mais loin de moi l'idée de le vérifier ici. Ce qui m'a paru plus intéressant, c'est de partir au plus près des réflexions de Sartre sur le néant.

\subsection{La structuration du champ perceptif}

Dans les premières pages consacrées à une phénoménologie du néant, Sartre ne se réfère pas d'abord au pouvoir de décision du sujet. En réalité, une des premières occurrences du néant concerne l'expérience d'un individu qui pénètre en retard dans un café à la recherche de Pierre avec lequel il a rendezvous $^{2}$. Cet exemple joue un rôle crucial dans l'argumentation sartrienne : il s'agit d'insister sur le fait que l'expérience du néant n'a pas son siège privilégié dans le jugement, comme on pourrait le croire. Le néant est d'abord et avant tout une expérience antéprédicative, ici en l'occurrence perceptive, ou comme dit Sartre, « intuitive ». Autrement dit, l'expérience que nous faisons du néant n'est pas d'emblée un acte de l'esprit, à savoir l'expérience cognitive d'un jugement assertorique négatif ( $" \mathrm{p} n$ 'est pas q »). C'est une expérience vécue, je fais l'expérience du néant d'une certaine manière « en chair et en os ». Ce qui, a priori, apparait comme une contradiction ou une hallucination (donc percevoir quelque chose qui n'existe pas), se révèle à l'analyse tout à fait exact

Lorsque j'entre dans ce café, pour y chercher Pierre, il se fait une organisation synthétique de tous les objets du café en fond sur quoi Pierre est donné comme devant paraître. Et cette organisation du café en fond est une première néantisation $^{3}$.

La première forme de néantisation est un effet de structure ou de structuration de mon champ perceptif: il y a là une démarcation qui se dessine entre le premier-plan de mon attente (Pierre) et l'arrière-plan du contexte dans lequel je me trouve. Mon attente, à savoir « apercevoir Pierre dans le café », repousse

${ }^{1}$ M. Heidegger, Être et Temps, trad. F. Vezin, Paris, Gallimard, 1998, § 60.

2 J.-P. Sartre, L'Être et le Néant, Paris, Gallimard, 1996, p. 43 sq.

${ }^{3}$ Ibid., p. 44. 
à l'arrière-plan toutes les personnes et les objets qui peuplent ce café ; ils sont certes toujours là, mais affectés d'un coefficient négatif, à savoir « tout ce qui n'est pas Pierre»; ils m'apparaissent précisément comme «n'étant pas Pierre ».

Notons cependant que cette première forme de néantisation est toutefois nécessaire à l'apparaître de Pierre. En effet, si je veux pouvoir identifier quelque chose dans mon horizon, encore faut-il que certaines choses se retirent, pour parler comme Heidegger, à l'arrière-plan — sans quoi j'aurais plutôt affaire à un chaos de données sensibles. Cette première forme de néantisation désigne donc la polarisation de mon champ perceptif en un premier-plan et arrière-plan. Suite à quoi je puis faire l'expérience d'une seconde forme de néantisation, à savoir l'absence de Pierre. Considérons la suite du passage :

Mais justement Pierre n'est pas là. [...] En fait Pierre est absent de tout le café ; son absence fige le café dans son évanescence, le café demeure fond, il persiste à s'offrir comme totalité indifférenciée à ma seule attention marginale, il glisse en arrière, il poursuit sa néantisation. Seulement il se fait fond pour une forme déterminée, il la porte partout au-devant de lui, il me la présente partout et [...] c'est précisément un évanouissement perpétuel, c'est Pierre s'enlevant comme néant sur le fond de néantisation du café. De sorte que ce qui est offert à l'intuition, c'est un papillotement de néant, c'est le néant du fond, dont la néantisation appelle, exige l'apparition de la forme, et c'est la forme — néant qui glisse comme un rien à la surface du fond. Ce qui sert de fondement au jugement : «Pierre n'est pas là », c'est donc bien la saisie intuitive d'une double néantisation ${ }^{1}$.

Autrement dit, le perpétuel mouvement de retrait du café invite, en vertu de la polarisation perceptive que j'ai soulignée, à l'émergence d'une figure qui ne vient pas. Non-advenue dont je fais bien l'expérience en chair et en os : avant de juger, je constate l'absence de Pierre, une absence dont Sartre dit qu'elle « hante ce café » et qu'elle est " la condition de son organisation néantisante [du café] en fond $»^{2}$. Aussi faut-il dire que c'est la valeur de la présence de Pierre qui détermine cette configuration perceptive particulière - et c'est bien la nature de la valeur, selon Sartre, que de « hanter $»^{3}$ l'être.

\footnotetext{
${ }^{1}$ Ibid.

2 Ibid., p. 45.

${ }^{3}$ Ibid., p. 129-130.
} 


\subsection{La liberté comme polarisation}

Que peut-on retenir de la précédente analyse ? Contrairement à un certain prêtà-penser sartrien, qui fait de la liberté et du néant des propriétés intrinsèques d'une subjectivité qui se constitue en face du monde, il faut dire que la liberté et le néant sont d'abord des propriétés de cette totalité constituée par le couple moi-monde. Autrement dit, la liberté sartrienne n'apparaît pas d'abord comme un « pouvoir discrétionnaire sur soi $»^{1}$ que nous fuirions dans la mauvaise foi — dont parle par exemple Romano.

Contrairement à l'idée d'un " pouvoir discrétionnaire sur soi », le pouvoir de néantisation nous apparaît ici, d'abord et en premier lieu, comme ces trous, ces creux et ces béances au sein de ma situation. Ce sont en réalité tous ces « vides » que L'Être et le Néant oppose régulièrement aux « pleins » de l'être-en-soi. Certes ces vides dans le réel sont le fruit d'une présence humaine : en effet, pour qu'il y ait néant, encore faut-il qu'il y ait sujet en relation avec le monde ; pour que je puisse apercevoir l'absence de Pierre, encore fautil que je l'aie attendu, que sa présence soit désirée, c'est-à-dire que j'aie projeté des possibilités, par définition néantisantes, à partir de ma situation.

On devrait probablement rappeler encore ici l'intentionnalité à vide de Husserl qui, bien qu'elle ne trouve pas nécessairement son remplissement, signifie encore quelque chose. Il semble, au contraire, que chez Sartre ce nonremplissement par l'intuition donatrice soit encore une perception de quelque chose, mais paradoxalement une perception du vide, une intuition de l'absence, du rien. Quoi qu'il en soit de la riche intertextualité qui traverse ce passage, le plus important à mes yeux, c'est que la liberté n'est pas une « idée de la raison ». La liberté se caractérise plutôt par ces écarts et ces interstices de néant dans mon expérience, ces vides au cœur du plein qui, comme l'ont enseigné les gestaltistes, sont nécessaires à la structuration de ma situation.

C'est pourquoi il faudrait se garder de faire de la transcendance du poursoi le lieu de la liberté. La liberté, c'est au contraire l'écart institué par la réalité-humaine en tant qu'elle n'est pas simplement au monde, mais rapport au monde ou «présence à soi » ${ }^{2}$, comme dit Sartre. La liberté c'est précisément cet effet de polarisation qui fait advenir l'écart entre le pour-soi et l'en-soi,

\footnotetext{
${ }^{1}$ C. Romano, « Sincérité et naturel » dans J. Michel, M. Gonçalo, P. Jesus [dir.], Du moi au soi. Variations phénoménologiques et herméneutiques, Rennes, PUR, 2017, p. 27 : « Pour Sartre, la mauvaise foi est une fuite devant une liberté tenue pour infinie et inconditionnelle et caractérisée comme liberté de se choisir ».

${ }^{2}$ J.-P. Sartre, L’Être et le Néant, op. cit., p. 109 sq.
} 
c'est-à-dire une relation intentionnelle ${ }^{1}$. C'est pourquoi la liberté n'est pas un «pouvoir discrétionnaire sur soi» mais au contraire, selon l'expression de Sartre, une « présence à soi », qu'il faut entendre paradoxalement, ou dialectiquement, comme une non-coïncidence à soi, comme une fissure invisible :

Au contraire, la présence à soi suppose qu'une fissure impalpable s'est glissée dans l'être. S'il est présent à soi, c'est qu'il n'est pas tout à fait soi. La présence est une dégradation immédiate de la coïncidence, car elle suppose la séparation. Mais si nous demandons à présent : qu'est-ce qui sépare le sujet de lui-même, nous sommes contraints d'avouer que ce n'est rien².

En d'autres termes, si Federer est bien ce champion de tennis suisse aux vingt titres du Grand Chelem, il ne l'est cependant pas comme cette table est une table ou ce stylo un stylo, c'est-à-dire dans un pur et simple rapport d'identité, sans négativité ou différence. Il se pourrait bien qu'on apprenne par la suite qu'il avait triché, ce qui, comme dans le cas d'Armstrong, donnerait un tout autre sens à ses exploits...

\subsection{Liberté, volonté et émotions}

À mon sens, cette conception de la liberté est la seule manière de rendre correctement compte de certaines propositions sartriennes sur les émotions. Ainsi, lorsque Sartre soutient que les émotions relèvent de la liberté du sujet, il ne veut pas dire que nous choisissons nos émotions, ni même que nous serions toujours libres à l'égard de celles-ci. Les émotions, tout comme la connaissance ou la volonté, sont des modes intentionnels et par conséquent relèvent de cet écart institué par le néant au sein de l'être que Sartre appelle aussi « liberté originelle $»^{3}$ ou « liberté protéiforme $»^{4}$.

\footnotetext{
${ }^{1}$ Cette lecture me semble trouver un argument de poids dans la position non-égologique défendue par Sartre dans La Transcendance de l'Ego. Continuant sur les traces de la cinquième des Recherches logiques de Husserl, Sartre fait de la conscience un événement relativement impersonnel, c'est-à-dire, dans mes termes, l'advenue d'une relation intentionnelle qui ne nécessite pas de postuler une quelconque réalité égologique en deçà d'elle (le temps du flux de conscience se chargeant de l'unification) et dont la liberté serait une propriété.

${ }^{2}$ Ibid., p. 113.

${ }^{3}$ Ibid., p. 488.

${ }^{4}$ M. Merleau-Ponty, Phénoménologie de la perception, Paris, Gallimard, 2013, p. 419.
} 
Cette liberté est en effet « protéiforme », non pas au sens où elle serait un plein pouvoir sur soi, mais au sens où nous disposons d'une multitude de manières de nous situer, c'est-à-dire de répondre aux exigences de la situation: la connaissance, la volonté, l'action ou les émotions sont autant de modes, de variations autour de cette « liberté originelle », c'est-à-dire autant de modes de vivre dans l'écart institué par la relation intentionnelle :

La liberté n'est rien d'autre que l'existence de notre volonté ou de nos passions, en tant que cette existence est néantisation de la facticité, c'est-à-dire d'un être qui est son être sur le mode d'avoir à l'être'.

De manière tout à fait conséquente, si Sartre situe l'émotion au même niveau que la volonté, c'est-à-dire comme une forme de liberté, c'est parce que l'une et l'autre représentent des possibilités de répondre à la situation : d'un côté nous agissons rationnellement et techniquement (selon une sorte de rationalité instrumentale), de l'autre nous le faisons magiquement, mais non moins « librement » puisque nous pouvons modifier la couleur des exigences qui pèsent sur nous, c'est-à-dire agir sur le sens que revêt la situation pour nous.

Soit par exemple la colère éprouvée devant ce clou qui ne s'enfonce pas comme il devrait. Cette colère vient répondre au « coefficient d'adversité des choses $»^{2}$, en quelque sorte « librement », c'est-à-dire à la faveur de l'écart entre moi et le clou; ce qui fait que je ne suis jamais face à de pures et simples contraintes physiques, même lorsque je dois me rendre à l'évidence que le mur résiste au clou. Cette résistance n'est jamais simplement de l'ordre d'une force physique, mais en même temps toujours de l'ordre d'une exigence qui peut être contournée comme retournée "magiquement», c'est-à-dire une résistance dont le sens ou l'urgence peuvent faire l'objet d'une modification de ma part. Pour le dire simplement, le clou et le mur représentent une contrainte physique toujours déjà inscrite dans une relation intentionnelle. Ainsi, en m'énervant, je constitue le mur en obstacle ou comme un ennemi à vaincre, alors qu'il n'avait été jusqu'à présent que le support matériel de mon activité pratique.

Malgré ces quelques précautions, il me semble toutefois difficile de penser que nous choisissons librement de répondre techniquement ou magiquement, comme si la réalité se laissait imposer son sens du dehors. Je pense que dans ce genre d'affirmation Sartre perd un peu de ce sens dialectique qu'il manifeste au début de L'Être et le Néant, lorsqu'il entrevoit la liberté comme

${ }^{1}$ Ibid.

${ }^{2}$ Ibid., p. 364. 
une force polarisante plutôt que comme un fiat du sujet, comme le laissent parfois suggérer certains passages de la quatrième partie.

\section{Les pathologies de la liberté}

Venons-en donc maintenant à la pathologie qu'une telle liberté saurait souffrir. La première idée qui nous vient à l'esprit, me semble-t-il, lorsqu'on s'en tient à la pensée de Sartre, c'est évidemment la mauvaise foi. Il y aurait dans la mauvaise foi, en effet, une forme d'aliénation inévitable de la condition humaine, une liberté qui feindrait de s'ignorer. J'aimerais toutefois porter mon attention sur un autre phénomène, plus discret, à savoir l'empâtement.

\subsection{Empâtement et mauvaise foi}

Sans doute l'empâtement peut être considéré comme une forme de mauvaise foi. N'est-ce pas le cas du garçon de café, qui se laisse enfermer dans son rôle, qui se fait chose garçon-de-café, qui, donc, s'empâte dans son rôle?

Pourtant, à y regarder de plus près, il faudrait se garder de faire de l'empâtement une simple métaphore pour la réification. D'ailleurs, l'expression ne se retrouve pas dans le chapitre sur la mauvaise foi, sinon peut-être de manière allusive lorsque Sartre évoque la tristesse dans laquelle mon corps paraît, en effet, s'empâter :

Elle [la tristesse] est le sens de ce regard terne que je jette sur le monde, de ces épaules voûtées, de cette tête que je baisse, de cette mollesse de tout mon corps $^{1}$.

L'idée même d' « empâtement» revient en réalité explicitement lorsqu'il est question du désir et plus spécifiquement du désir sexuel dans le chapitre sur les « relations concrètes avec autrui ».

\subsection{Empâtement et désir}

Le désir, en tant que tel, n'a rien pour Sartre d'empâté ou d'empâtant. Comme l'a souligné Bernet dans son étude sur Sartre ${ }^{2}$, le désir apparaît d'emblée dans

${ }^{1}$ Ibid., p. 95.

${ }^{2}$ R. Bernet, Conscience et existence, Paris, PUF, 2004, p. 171 sq. 
la deuxième partie de L'Être et le Néant comme une forme de néantisation, à condition qu'on ne l'aplatisse pas sur un simple conatus pulsionnel. Il s'agit en réalité du désir d'autofondation de la conscience, soutient Bernet ${ }^{1}$. Or, le thème du désir disparaît ensuite des propos de Sartre pour revenir, bien plus tard, dans le chapitre sur «les relations concrètes avec autrui » ${ }^{2}$, mais cette fois-ci sous la forme plus spécifique du désir sexuel - des passages que Bernet ne semble pas avoir retenus dans son analyse.

Pour Sartre, l'affaire semble entendue. Le désir est une forme de conscience intentionnelle, au sens où l'on désire toujours quelque chose, « $\mathrm{p} »$ ou « que $\mathrm{p}$ ». Pourtant, lorsqu'il est orienté sur autrui, ce désir devient une conscience « trouble »" comme il le souligne aux mêmes pages. Il faut comprendre par-là que la visée, l'éclatement de la conscience dans le désir sexuel, n'est pas aussi limpide et immédiate que dans un autre acte intentionnel, comme la faim par exemple. Autant la sensation de faim possède une forme « empruntée d'intentionnalité » (borrowed intentionality), selon l'expression heureuse de Peter Goldie $^{4}$, autant dans le désir la conscience semble perdre de cette transparence intentionnelle. Pour le dire dans un vocabulaire sartrien, dans le désir, la conscience apparaît comme freinée dans son dépassement ou son éclatement vers le monde.

Selon Sartre, le désir sexuel trouble et freine la conscience dans son dépassement, parce qu'elle s'y trouve soudain retenue par le corps. Dans le désir sexuel, les sensations corporelles ne paraissent plus pouvoir refluer dans l'arrière-plan de nos activités, ou s'intégrer dans un projet corporel global, comme c'est le cas dans la faim ou n'importe quel autre de nos élans vitaux :

Dans le désir sexuel, certes, on peut retrouver cette structure commune à tous les appétits : un état du corps. [...] Et la conscience désirante existe cette facticité ; c'est à partir d'elle - nous dirions volontiers : à travers elle — que le corps désiré apparaît comme désirable. Toutefois, si nous nous bornions à le décrire ainsi, le désir sexuel apparaîtrait comme un désir sec et clair, comparable au désir de boire et de manger. Il serait fuite pure de la facticité vers d'autres possibles. Or chacun sait qu'un abîme sépare le désir sexuel des autres appétits. [...] C'est qu'on ne désire pas une femme en se tenant tout entier hors du désir, le désir me compromet ; je suis complice de mon désir. Ou plutôt le désir est tout entier chute dans la complicité avec le corps. Il n'est pour chacun

\footnotetext{
${ }^{1}$ Ibid., p. 176.

${ }^{2}$ J.-P. Sartre, L’Être et le Néant, op. cit., p. 425 sq.

${ }^{3}$ Ibid., p. 427.

${ }^{4}$ P. Goldie, « Emotions, feelings and intentionality », dans Phenomenology and the Cognitive Sciences n³ (2002/1), p. 247.
} 
que de consulter son expérience : on sait que dans le désir sexuel la conscience est comme empâtée, il semble qu'on se laisse envahir par la facticité, qu'on cesse de la fuir et qu'on glisse vers un consentement passif au désir. [...] La conscience alourdie et pâmée glisse vers un alanguissement comparable au sommeil ${ }^{1}$.

Il y aurait beaucoup à dire sur ce passage, mais je relèverai surtout deux idées centrales :

1. Le désir est une "chute dans la complicité avec le corps »; on a là une définition de l'empâtement qui pourrait indiquer, dans la perspective sartrienne, une sorte de pathologie de la liberté.

2. Cette chute ne peut se réaliser qu'à la faveur d'un consentement au corps. Parce que le désir sexuel n'est en effet ni un acte volontaire ni non plus un simple arc réflexe, il nécessite qu'on y consente, i.e., comme pour le sommeil, qu'on se laisse aller à l'alanguissement de la chair.

Contrairement à certaines analyses intellectualistes en philosophie de l'esprit, Sartre ne considère pas que le désir est un simple état mental, dont l'occurrence activerait par la suite des régions du corps objectif (pouls, alanguissement, tension, etc.). Le désir n'est pas pensée, mais il est caresse. La caresse porte le désir comme le signe linguistique supporte la pensée ${ }^{2}$. Dans le désir c'est tout le corps propre qui se porte de manière significative en direction d'autrui. Il ne s'agit donc ni d'un pur état mental ni d'une simple augmentation des constantes physiologiques.

Toutefois, selon Sartre, l'intentionnalité de la caresse ne ressemble en rien aux formes pratiques d'intentionnalité corporelle, telle que par exemple un geste de la main pour héler un taxi. Dans le geste, les sensations du corps sont dépassées et traversées en direction du monde : comme dans la préhension, on agit dans et sur le monde. Dans la caresse, au contraire, mon corps s'empâte (ou je me laisse empâter) dans ses sensations, au lieu de se retirer à l'arrière-plan. La caresse n'est donc pas une simple extase pratique vers le dehors, mais un consentement à la passivité :

Dans la caresse, ce n'est pas mon corps comme forme synthétique en action qui caresse autrui : mais c'est mon corps de chair qui fait naître la chair d'autrui. La caresse est faite pour faire naître par le plaisir le corps d'autrui à autrui et à moi-même comme passivité touchée dans la mesure où mon corps se fait chair pour le toucher avec sa propre passivité, c'est-à-dire en se caressant à lui plutôt qu'en le caressant. C'est pourquoi les gestes amoureux ont une langueur qu'on

${ }^{1}$ J.-P. Sartre, L'Être et le Néant, op. cit., p. 427-428.

${ }^{2}$ Ibid., p. 430. 
pourrait presque dire étudiée : il ne s'agit pas tant de prendre une partie du corps de l'autre que de porter son propre corps contre le corps de l'autre. Non pas tant de pousser ou de toucher, au sens actif, mais de poser contre ${ }^{1}$.

Il me semble, sans vouloir surinterpréter, qu'on retrouve ici une variation autour du thème fameux de la main touchante-touchée de Husserl ${ }^{2}$. Sauf que chez Sartre le thème est transposé du champ cognitif au champ affectif, de sorte que le contact des épidermes renvoie au jeu érotique des corps : la caresse est à la fois un empâtement de ma liberté dans la chair tout comme elle est une invitation à l'empâtement d'autrui dans la sienne.

Il faut en même temps rappeler que cette description prend place au cœur des relations avec autrui. Autrement dit, le désir sexuel est à comprendre comme une manière de répondre au regard de l'autre. Il s'agit, dans le désir sexuel, de résoudre le conflit des regards, mais en quelque sorte au niveau de l'en-soi. Il s'agit d'amener l'autre à rendre les armes en commençant par se rendre vulnérable à ses yeux dans l'empâtement. Les regards cèdent ici la place à la chair, assurant une coïncidence avec soi et avec l'autre, coïncidence qui semblait impossible au départ des analyses de L'Être et le Néant.

C'est pourquoi cette analyse apparaît somme toute parfaitement cohérente avec la position de Sartre sur la liberté. L'empâtement, c'est en réalité un colmatage des trous, des vides que la liberté avait généré au sein de l'être, et dont le regard d'autrui est une forme exemplaire - souvenons-nous que ce regard est précisément pour Sartre un trou, un vide, dans mon champ perceptif, cette « hémorragie $»^{3}$ que je ne pourrai jamais stopper. Dans le désir sexuel je cherche par conséquent à faire disparaître la béance que le regard d'autrui a introduite dans mon monde. En me faisant chair, j'entraîne l'autre sur le versant de l'en-soi, je réalise l'unité avec moi et avec l'autre sur ce terrain-là, c'est-à-dire au sein de la plénitude de nos matérialités respectives.

Quelques lignes plus loin, Sartre généralise cette analyse de l'empâtement à tout le rapport au monde, qui n'est pas sans nous faire penser à la « chair du monde $»^{4}$ de Merleau-Ponty :

Un contact est caresse, c'est-à-dire que ma perception n'est pas utilisation de l'objet et dépassement du présent en vue d'une fin ; mais percevoir un objet, dans l'attitude désirante, c'est me caresser à lui. Ainsi suis-je sensible, plus qu'à la forme de l'objet et plus qu'à son instrumentalité, à sa matière (grumeleuse,

\footnotetext{
${ }^{1}$ Ibid., p. 431.

${ }^{2}$ E. Husserl, Idées directrices II, trad. É. Escoubas, Paris, PUF, 1996, § 36.

${ }^{3}$ J.-P. Sartre, L'Être et le Néant, op. cit., p. 294 sq.

${ }^{4}$ M. Merleau-Ponty, Le Visible et l'Invisible, Paris, Gallimard, 2004, p. 176 sq.
} 
lisse, tiède, graisseuse, rêche, etc.) et je découvre dans ma perception désirante quelque chose comme une chair des objets. Ma chemise frotte contre ma peau et je la sens : elle qui d'ordinaire est pour moi l'objet le plus lointain devient le sensible immédiat, la chaleur de l'air, le souffle du vent, les rayons du soleil, etc., tout m'est présent d'une certaine manière, comme posé sans distance sur moi et révélant ma chair par sa chair. De ce point de vue, le désir n'est pas seulement l'empâtement d'une conscience par sa facticité, il est corrélativement l'engluement d'un corps par le monde ; et le monde se fait engluant; la conscience s'enlise dans un corps qui s'enlise dans le monde ${ }^{1}$.

Je ne sais pas s'il faut parler de l'empâtement comme d'une forme de pathologie de la liberté. Peut-être qu'en un sens, c'est bien le cas ; peut-être qu'il faudrait déplorer l'attitude de ces sujets qui renoncent à assumer l'écart et la fissure qui caractérisent notre présence au monde et qui s'abandonnent ainsi, lascivement, à l'en-soi. Mais, pour terminer, j'aimerais pour ma part esquisser quelques remarques dans une autre direction.

\section{L'empâtement et la psychopathologie de l'anorexie}

Il me semble, modestement parlant, que ces analyses présentent des ressources précieuses pour une psychopathologie clinique qui cherche à comprendre comment une présence au monde peut être, selon l'expression de Binswanger, manquée ou malheureuse (missglückte) ${ }^{2}$.

Comme parfois chez Sartre, il y a, dans cette analyse de l'érotique, une généralisation abusive d'un type particulier de rapport au monde, à soi et aux autres - comme l'avait montré Merleau-Ponty à propos du regard aliénant d'autrui. De même ici, il est possible que cet empâtement relève finalement plus de l'expérience pathologique du corps et du désir que de l'expérience normale.

Je ne veux pas dire par là que la relation sexuelle est toujours simple et de l'ordre d'une danse harmonieuse des corps. Ce serait naïf. Toutefois, il me semble que toute cette description sartrienne se fait encore trop du point de vue d'un spectateur étranger. Je veux dire que, lorsque Sartre parle d'empâtement, il se place en réalité en surplomb, il adopte le point de vue du philosophe qui observe à distance un comportement érotique, c'est-à-dire précisément sans y consentir. C'est pourquoi il y découvre moins l'authenticité de l'engagement

${ }^{1}$ J.-P. Sartre, L'Être et le Néant, op. cit., p. 432.

${ }^{2} \mathrm{~L}$. Binswanger, Trois formes manquées de la présence humaine, trad. J.-M. Froissart, Paris, Le Cercle Herméneutique, 2002. 
qu'une comédie, une simagrée, celle de la " langueur de ces gestes étudiés » dont il est question dans l'une des citations précédentes. J'ai l'impression, mais je me trompe peut-être, que l'analyse du désir est toujours faite en comparaison avec le sujet pratique qui manipule le monde ; de sorte que le désir ne peut apparaître que comme une forme dégradée de l'action sur l'environnement, une « chute ${ }^{1}$, comme il le dit, de notre pouvoir sur le monde vers la matérialité de l'en-soi. Pour le dire plus simplement, pourquoi faire du consentement à la chair du monde nécessairement une forme d'empâtement au lieu d'y voir, un peu à la manière de Merleau-Ponty, une entrée en sympathie avec l'environnement et avec autrui ?

L'expérience de l'empâtement ressemble en réalité, à s'y méprendre, à l'expérience que l'anorexique peut avoir de son corps et de son rapport charnel au monde. C'est à mon avis toute la richesse de cette analyse, à savoir de nous introduire au cœur d'un rapport pathologique au(x) corps. L'anorexique, mais ce n'est probablement de loin pas la seule, vit son lien charnel avec le monde, avec les nourritures, avec autrui ainsi qu'avec son corps, précisément sur le mode de la compromission, voire de contamination (par la chair du monde). Aussi, ce que Sartre appelle, de l'extérieur, l'empâtement pourrait tout aussi bien désigner, de l'intérieur, notre animation corporelle, animation sur laquelle la philosophe américaine Maxine Sheets-Johnstone a régulièrement insisté dans son analyse du contact charnel et de la matérialité :

Quand nous faisons l'expérience d'une chose en profondeur plutôt que comme une forme reconnaissable mais vague sur laquelle notre regard ne fait que passer — nous laissons notre sensibilité de surface venir au premier plan. Ce faisant, nous nous ouvrons à la possibilité d'être touché et é-mu (being moved) par ce qu'il y a. Réciproquement, nous découvrons la possibilité de sonder (fathoming) la densité d'un autre vivant ou d'une chose. Nous laissons une certaine forme nous transporter de la surface de notre sensibilité à la densité de la chair. Nous nous ouvrons à de nouvelles possibilités d'accord (attunement) ${ }^{2}$.

L'anorexique ne connaît plus cette animation, cet élan vital, mais elle fait au contraire l'expérience de son empâtement. Elle se refuse au compromis avec la chair, elle se refuse au désir, à l'appétit et à la sexualité. Elle trouve au contraire refuge dans l'attitude volontariste, spirituelle et active, à laquelle Sartre semble reconnaître, du moins dans certains passages, une forme insigne de liberté. Si l'anorexique éprouve du plaisir, c'est dans la maîtrise et le contrôle

\footnotetext{
${ }^{1}$ Voir la citation de Sartre, supra, p. 7.

${ }^{2}$ M. Sheets-Johnstone, The Corporeal Turn, Charlottesville, Imprint Academic, 2009, p. 144 .
} 
qu'elle peut exercer sur elle et sur son monde. Ce dont souffre l'anorexique, et ce n'est certainement pas la seule, c'est bien de l'impossibilité de consentir au corps, de lui laisser quelque peu l'initiative, comme dans l'appétit (mais aussi dans l'endormissement). Son corps n'est précisément plus, comme l'a bien souligné Sartre, cette matrice transparente qui favorise notre intégration au milieu, mais une chose-chair, cette conscience troublée qui fait écran entre moi et le monde.

La transcendance corporelle est ici freinée dans son élan et s'abîme dans ses sensations. La matière se présente au sujet sous forme de pâte, c'est-à-dire comme des paquets de qualités et de sensations abstraites (grumeleuse, lisse ou, dans le cas particulier de l'anorexique, épaisse, lourde, grasse). Là où le sujet consentant à la sensibilité percevrait les valeurs vitales qui hantent les choses, c'est-à-dire qui organisent les qualités sous forme d'unités de sens motivationnelles (des affordances), l'anorexique paraît éprouver de manière écrasante le trop-plein de sa chair et de la chair du monde. C'est pourquoi la nourriture n'invite plus chez elle à la jouissance, mais elle réveille ces mêmes sensations qui passent chez nous au second plan (sensations de déglutition, de digestion, de satiété, etc.). Aussi l'appétit, la jouissance et l'élan vers l'assimilation du monde sont-ils remplacés chez elle par la maîtrise pratique, le contrôle, le monitoring psychique, comme pour restaurer une liberté en train de lui échapper.

Il faut par conséquent revenir de la psychopathologie vers Sartre, pour soutenir que l'empâtement, cette forme dégradée de conscience, cette « chute dans la complicité avec le corps ", n'est que l'envers d'une forme authentique d'animation corporelle, de vitalité - celle-là même que l'anorexique semble avoir perdue chemin faisant. 